\begin{tabular}{|c|l|}
\hline Title & Multistate image restoration by transmission of bit-decomposed data \\
\hline Author(s) & Tadaki, Takashi; Inoue, Jun-ichi \\
\hline Citation & $\begin{array}{l}\text { Physical Review E, 65(1),016101 } \\
\text { https://doi.org/10.1103/PhysRevE.65.016101 }\end{array}$ \\
\hline Issue Date & 2001-12-04 \\
\hline Doc URL & http://hdl.handle.net/2115/5807 \\
\hline Rights & Copyright $\odot 2002$ A merican Physical Society \\
\hline Type & article \\
\hline File Information & PRE65.pdf \\
\hline
\end{tabular}

Instructions for use 


\title{
Multistate image restoration by transmission of bit-decomposed data
}

\author{
Takashi Tadaki \\ Department of Physics, Tokyo Institute of Technology, Oh-okayama, Meguro-ku, Tokyo 152-8551, Japan \\ Jun-ichi Inoue \\ Complex Systems Engineering, Graduate School of Engineering, Hokkaido University, N13-W8, Kita-ku, Sapporo 8628, Japan
}

(Received 20 May 2001; revised manuscript received 8 August 2001; published 4 December 2001)

\begin{abstract}
We report on the restoration of gray-scale image when it is decomposed into a binary form before transmission. We assume that a gray-scale image expressed by a set of $Q$-Ising spins is first decomposed into an expression using Ising (binary) spins by means of the threshold division, namely, we produce $(Q-1)$ binary Ising spins from a $Q$-Ising spin by the function $F\left(\sigma_{i}-m\right)=1$ if the input data $\sigma_{i} \in\{0, \ldots, Q-1\}$ is $\sigma_{i} \geqslant m$ and 0 otherwise, where $m \in\{1, \ldots, Q-1\}$ is the threshold value. The effects of noise are different from the case where the raw $Q$-Ising values are sent. We investigate whether it is more effective to use the binary data for transmission, or to send the raw $Q$-Ising values. By using the mean-field model, we analyze the performance of our method quantitatively. In order to investigate what kind of original picture is efficiently restored by our method, the standard image in two dimensions is simulated by the mean-field annealing, and we compare the performance of our method with that using the $Q$-Ising form. We show that our method is more efficient than the one using the $Q$-Ising form when the original picture has large parts in which the nearestneighboring pixels take close values.
\end{abstract}

DOI: 10.1103/PhysRevE.65.016101

PACS number(s): 02.50.-r, 05.20.-y, 05.50.+q

\section{INTRODUCTION}

Statistical-mechanical approaches to the problems in information science are employed frequently, and it is now known that such methods are very useful in various problems [1]. Among such problems, the image restoration problem has been investigated both theoretically and practically [2-4]. We usually send and receive the information by means of various networks. The information is the data of document, sound, picture, and so on. However, it is generally impossible for a receiver to receive complete transmitted data when a sender transmits something, because the data are transmitted through a noisy channel. If we restrict the type of data to that of the picture, the original image is affected by some kind of noise when it is sent by a defective fax, a fickle email, etc. When we receive such a corrupted image, we have to convert it using some kind of filter to obtain the original image. Basically, it is the image restoration problem to estimate the original data (the original image) from the received, corrupted data (the degraded image). We may regard the digital picture as a discrete spin system. For example, a black and white image corresponds to an Ising spin system by identifying the white color with +1 and the black with -1 . Furthermore, the theory of image restoration is constructed by considering that two axes of the plane, the $x$ and $y$ axis, correspond to two axes of time in the stochastic process. That is, the Markov process that the event occurs at a specific time is affected by what happened at neighboring pixels at a one-time step before.

There are mainly two standard approaches to the image restoration by means of the method of statistical mechanics. One is called the maximum a posteriori (MAP) estimation, in which the estimation of the original image is given by maximizing a posterior probability distribution. This estimation will be seen to correspond to a search of the ground state of a spin system described by the effective Hamiltonian in the context of statistical mechanics. Another is the estimation in which we regard the expectation value with respect to the maximized marginal posterior probability at each site in thermal equilibrium as the original image and is called the maximum posteriori marginal (MPM) estimation. This estimation is also called the finite-temperature restoration. Therefore, the MPM estimation includes the MAP estimation. Among the two estimations, it was proposed by Marroquin et al. [5] that the MPM estimation gives better performance than the MAP estimation. Nishimori and Wong [6] proved this fact for black and white images using a rigorous inequality. With respect to the gray-scale image, the same has been shown by Tanaka [7] from a different viewpoint.

Not only black and white images but also gray-scale images are actively investigated by many people in the field of statistical mechanics. Restoration of gray-scale image using chiral Potts spin [9] may not be appropriate since chiral Potts spin cannot express the distance among different states. However, $Q$-Ising spin [8] may express the gray-scale level at least. Restoration of gray-scale image using $Q$-Ising spin was first investigated by Inoue and Carlucci [10].

Inspired by their studies, we investigate the restoration of the gray-scale image expressed by the $Q$-Ising spin when it is decomposed into the Ising spin before transmission. In this method, the gray-scale image is decomposed into binary data by means of the method of threshold division before it is transmitted. Then the bit-decomposed data is transmitted through a noisy channel. Therefore, the effects of noise are different from those in the $Q$-Ising form. We show how well the original image is restored in our method in comparison to the restoration using the $Q$-Ising form.

This paper is organized as follows. In the next section, we explain the general formulation of image restoration and the method of threshold division. In Sec. III, we analyze the 
static properties of image restoration in the infinite-range model. In Sec. IV, we verify the result of the infinite-range model in realistic pictures by means of Monte Carlo simulation. In Sec. V, standard image is restored by the method of mean-field annealing. The performance of restoration in our method is compared with that using the $Q$-Ising form. The final section is denoted to summary and discussions.

\section{GENERAL FORMULATION}

\section{A. Image restoration}

A gray-scale image is represented by a set $\{\xi\}_{i=1, \ldots, N}$ of fixed values. The variable $\xi_{i}$ takes an integer value between 0 and $Q-1$. Even in black and white image, in addition to gray-scale image, most of the natural images have nontrivial structures generally. Therefore, it is impossible to discuss the property of a given specific natural image, exactly. Nevertheless, we may notice that an eminent property of natural images is local smoothness. Therefore, we assume that the original image is generated by the Boltzmann probability represented by the following:

$$
P_{s}(\{\xi\})=\frac{1}{\mathcal{Z}\left(\beta_{s}\right)} \exp \left[-\frac{\beta_{s}}{2 z} \sum_{(i j)}\left(\xi_{i}-\xi_{j}\right)^{2}\right],
$$

where $(i j)$ represents interacting sites and $z$ is the coordination number. $Z\left(\beta_{s}\right)$ is the normalization constant, and $\beta_{s}$ $\left(=T_{s}^{-1}\right)$ is the inverse temperature to generate the original image. If $\beta_{s}$ is large, an original image with many clusters consisting of the same value is generated.

A degraded image is generated by sending data of the original image through a noisy channel. We consider two kinds of noise. One is the binary noise caused by a binary symmetric channel and another is the Gaussian noise caused by the Gaussian channel.

\section{Gaussian channel}

In the Gaussian channel, the output $\tau_{i}$ for an input $\xi_{i}$ is a Gaussian random variable with mean $\tau_{0} \xi_{i}$ and variance $\tau^{2}$. The probability distribution of output given the input $\{\xi\}$ is written as

$$
P(\{\tau\} \mid\{\xi\})=\frac{1}{\sqrt{2 \pi} \tau} \exp \left[-\frac{1}{2 \tau^{2}} \sum_{i}\left(\tau_{i}-\tau_{0} \xi_{i}\right)^{2}\right] .
$$

The degraded image $\{\tau\}_{i=1, \ldots, N}$ is generated by this probability distribution.

According to the Bayes formula, the posterior probability $P(\{\sigma\} \mid\{\tau\})$ that the estimate of source sequence, namely, the restored image, is $\{\sigma\}_{i=1, \ldots, N}$, provided that the output is $\{\tau\}$, is given as

$$
\begin{aligned}
P(\{\sigma\} \mid\{\tau\}) & =\frac{P(\{\tau\} \mid\{\sigma\}) P_{m}(\{\sigma\})}{\operatorname{tr}_{\{\sigma\}} P(\{\tau\} \mid\{\sigma\}) P_{m}(\{\sigma\})} \\
& \sim \exp \left(-h \sum_{i}\left(\sigma_{i}-\tau_{i}\right)^{2}-\frac{\beta_{m}}{2 z} \sum_{i j}\left(\sigma_{i}-\sigma_{j}\right)^{2}\right) \\
& \equiv \exp \left(-\mathcal{H}_{\mathrm{G}}\right) .
\end{aligned}
$$

Since we may use the degraded image only and do not know the other information of the original image, we introduced the model prior $P_{m}(\{\sigma\})$ to represent a priori knowledge on natural image: $P_{s}(\{\xi\})$ represented by Eq. (1). Furthermore, prior parameters $\beta_{s}$ and $\tau_{0} / \tau^{2}$ to control the generation of original image and degraded image, respectively, are unknown quantities. Accordingly, we have to use the so-called hyperparameters $\beta_{m}$ and $h$ instead of prior parameters $\beta_{s}$ and $\tau_{0} / \tau^{2}$. Then, controlling these hyperparameters, we may obtain the optimal restored image.

\section{Binary symmetric channel}

When binary data $\left\{\xi_{b}\right\}(Q=2)$ takes 0 or 1 , the type of noise may also be binary. In such a case, a pixel of the original image is flipped with the probability $p$, the error rate. The error probabilities of flipping the signal +1 to 0 and 0 to +1 are the same. The probability distribution of this output $\left\{\tau_{b}\right\} \in\{0,1\}$ is expressed as follows:

$$
P\left(\left\{\tau_{b}\right\} \mid\left\{\xi_{b}\right\}\right)=\frac{1}{Z_{\tau}} \exp \left[-\beta_{\tau} \sum_{i}\left(\tau_{i, b}-\xi_{i, b}\right)^{2}\right],
$$

where

$$
Z_{\tau}=\operatorname{Tr}_{\tau_{b}} \exp \left[-\beta_{\tau} \sum_{i}\left(\tau_{i, b}-\xi_{i, b}\right)^{2}\right]=\left[1+e^{\beta_{\tau}}\right]^{N} .
$$

The parameter $\beta_{\tau}$ is defined by $p$ becomes

$$
\beta_{\tau}=\ln \frac{1-p}{p} \text {. }
$$

The posterior probability in this case is

$$
\begin{aligned}
& P\left(\left\{\sigma_{b}\right\} \mid\left\{\tau_{b}\right\}\right)= \frac{P\left(\left\{\tau_{b}\right\} \mid\left\{\sigma_{b}\right\}\right) P_{m}\left(\left\{\sigma_{b}\right\}\right)}{\operatorname{tr}_{\sigma_{b}} P\left(\left\{\tau_{b}\right\} \mid\left\{\sigma_{b}\right\}\right) P_{m}\left(\left\{\sigma_{b}\right\}\right)} \\
& \sim \exp \left(-h \sum_{i}\left(\sigma_{i, b}-\tau_{i, b}\right)^{2}\right. \\
& \\
&\left.-\frac{\beta_{m}}{2 z} \sum_{i j}\left(\sigma_{i, b}-\sigma_{j, b}\right)^{2}\right) \\
& \equiv \exp \left(-\mathcal{H}_{\mathrm{B}}\right) .
\end{aligned}
$$

\section{B. MPM estimation and mean-square error}

Next, we discuss the method to estimate the original image from the preceding posterior probability distribution and to evaluate the restored image that is obtained by such estimations.

We consider the marginal distribution obtained from the posterior probability distribution Eq. (3) [or Eq. (6)] to estimate the original image

$$
\bar{P}\left(\sigma_{i} \mid\{\tau\}\right) \equiv \sum_{\sigma \neq \sigma_{i}} P(\{\boldsymbol{\sigma}\} \mid\{\boldsymbol{\tau}\})
$$


Using this marginal probability distribution, we calculate the local magnetization at a site $i$ as given by

$$
\left\langle\sigma_{i}\right\rangle_{\beta_{m}, h} \equiv \sum_{\sigma_{i}=0}^{Q-1} \sigma_{i} \bar{P}\left(\sigma_{i} \mid\{\tau\}\right) .
$$

Since the above expectation value is a continuous real number, we need to change it into an integer by means of the following function $\Omega$ : because the original image consists of the discrete value in digital images, the restored image is the estimation of original image should, properly, take the discrete value

$$
\begin{aligned}
\Omega\left(\left\langle\sigma_{i}\right\rangle_{\beta_{m}, h}\right) \equiv & \sum_{k=1}^{Q} k\left[\Theta\left(\left\langle\sigma_{i}\right\rangle_{\beta_{m}, h}-\frac{2 k-1}{2}\right)\right. \\
& \left.-\Theta\left(\left\langle\sigma_{i}\right\rangle_{\beta_{m}, h}-\frac{2 k+1}{2}\right)\right] .
\end{aligned}
$$

A real number $\left\langle\sigma_{i}\right\rangle_{\beta_{m}, h}$ is translated into the closest integer. Then we regard the above discrete value $\Omega\left(\left\langle\sigma_{i}\right\rangle\right)$ as the value of $i$ th pixel for restored image at finite $\beta_{m}$ and $h$ in the finite-temperature process.

In the MAP estimation, the estimation of the original image is regarded as the set $\left\{\sigma_{i}\right\}$ that maximizes the posterior probability distribution [Eqs. (3),(6)]. In other words, it is the set that minimizes the energy of the system described by the Hamiltonian $\mathcal{H}_{\mathrm{G}}$ or $\mathcal{H}_{\mathrm{B}}$ and is actually the ground state. Consequently, the above estimation corresponds to the MAP estimation when $T_{m} \rightarrow 0 \quad\left(\beta_{m} \rightarrow \infty\right)$, keeping $H=h / \beta_{m}$ constant.

It is very important to evaluate the performance of restoration by means of these estimations. For this purpose, we assume that we know the original image and evaluate the performance of restoration by measuring the distance between the original and the restored images given by the above estimation. In order to measure the distance between the original and the restored images, we use the following mean square error as the distance:

$$
H_{\mathrm{D}}=\frac{1}{N} \sum_{i}\left[\xi_{i}-\Omega\left(\left\langle\sigma_{i}\right\rangle_{\beta_{m}, h}\right)\right]^{2}
$$

whose value depends on the hyperparameters $h$ and $\beta_{m}$ appearing in the thermal average.

\section{Method of threshold division}

We next discuss the restoration of the gray-scale image using bit-decomposed data. It is expected that the effects of noise on the binary data is lower than that on the $Q$-value data for image restoration: the binary data is estimated easily compared with the $Q$-value data because of the explicit representation. Since the original image is estimated by using the information of degraded image, the performance of restoration ought to be deeply affected by the effects of noise. Therefore, we expect that the performance of restoration is improved by using the binary data instead of the $Q$-value data.

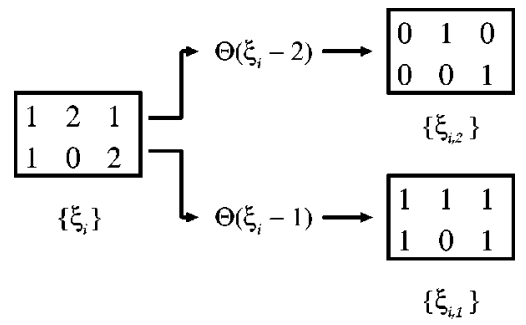

FIG. 1. The example of decomposition by means of the method of threshold division in the $Q=3$ and six-site case.

We generate binary data from the $Q$-value data using the $Q$-Ising form by means of the following function:

$$
\Theta\left(\xi_{i}-k\right)=\xi_{i, k}=1\left(\xi_{i} \geqslant k\right), \quad 0\left(\xi_{i}<k\right),
$$

where $k(\in\{1,2, \ldots, Q-1\})$ is the threshold value and $\xi_{i}$ $(\in\{0,1, \ldots, Q-1\})$ is the input data. We obtain $(Q-1)$ sets of binary data from a $Q$-value data by using this function with threshold value changed from 1 to $Q-1$. This method is called the threshold division. For example, we consider a set with $Q=3$ ( 0 , or 1 , or 2 ) and six pixels: $\{1,2,1,1,0,2\}$. If we insert this set into the above function with the threshold value $k=2$, the binary set $\{0,1,0,0,0,1\}$ is generated. The set $\{1,1,1,1,0,1\}$ is also generated when $k=1$. Thus, two binary sets $\{0,1,0,0,0,1\}$ and $\{1,1,1,1,0,1\}$ are generated from the three-value set $\{1,2,1,1,0,2\}$. These operations are depicted in Fig. 1. The expression of binary data $\xi_{i, k}(\in\{0,1\})$ produced by this method is different from the usual binary notation because the relation between a $Q$-value data and $(Q$ $-1)$ sets of binary data becomes

$$
\xi_{i}=\xi_{i, 1}+\xi_{i, 2}+\cdots+\xi_{i, Q-1}=\sum_{k=1}^{Q-1} \xi_{i, k}
$$

We call binary data generated by this threshold division the bit-decomposed data (BDD).

Next, the process in the restoration of gray-scale image using the bit-decomposed data becomes the following:

(1) Decompose a $Q$-value data (original image) into ( $Q$ -1) sets of binary data before transmission.

(2) Send $(Q-1)$ sets of binary data through a noisy channel.

(3) Receive $(Q-1)$ sets of corrupted binary data (degraded image).

(4) Restore the original image from the degraded image.

Obviously, this procedure is different from the method in which the raw $Q$-value data are sent.

Following the general formulation mentioned in the previous section, we formulate the above process. The posterior probability necessary for restoration is the following:

$$
\begin{aligned}
& P\left(\{\sigma\} \mid\left\{\tau_{1}\right\},\left\{\tau_{2}\right\}, \ldots,\left\{\tau_{Q-1}\right\}\right) \\
& \quad=\frac{P\left(\left\{\tau_{1}\right\},\left\{\tau_{2}\right\}, \ldots\left\{\tau_{Q-1}\right\} \mid\{\sigma\}\right) P_{m}(\{\sigma\})}{\operatorname{tr}_{\{\sigma\}} P\left(\left\{\tau_{1}\right\},\left\{\tau_{2}\right\}, \ldots\left\{\tau_{Q-1}\right\} \mid\{\sigma\}\right) P_{m}(\{\sigma\})} \\
& \quad \sim \exp \left(-h \sum_{i} \sum_{k}\left(\sigma_{i, k}-\tau_{i, k}\right)^{2}-\frac{\beta_{m}}{2 z} \sum_{i, j}\left(\sigma_{i}-\sigma_{j}\right)^{2}\right),
\end{aligned}
$$


where we used $\{\sigma\}$ to denote the dynamical variables used to estimate $\{\xi\}$. The notation $\sigma_{i, k} \in\{0,1\}$ is the estimation of decomposed data $\xi_{i, k}$. The notation $\tau_{i, k} \in\{0,1\}$ is the degraded binary data. The relation between $\sigma_{i, k}, \tau_{i, k}$, and $\sigma_{i}, \tau_{i}$ is the same as that between $\xi_{i}$ and $\xi_{i, k}$ [Eq. (12)].

In the restoration of gray-scale image using the $Q$-Ising form, the posterior probability distribution is given by

$$
P(\{\sigma\} \mid\{\tau\}) \sim \exp \left(-h \sum_{i}\left(\sigma_{i}-\tau_{i}\right)^{2}-\frac{\beta_{m}}{2 z} \sum_{i j}\left(\sigma_{i}-\sigma_{j}\right)^{2}\right) .
$$

The difference of the posterior probability between our method (13) and the $Q$-Ising form (14) is only in the randomfield term. That is to say, the effects of noise in our method are different from the case where the raw $Q$-Ising data are sent.

\section{ANALYSIS OF THE INFINITE-RANGE MODEL}

In this section, we discuss the restoration of the gray-scale image in the infinite-range model (mean-field model). Since the infinite-range model is the model in which all pixels interact mutually, it is not useful for restoration of real images directly. However, the analysis of image restoration in the infinite-range model is very useful in understanding qualitatively the property of macroscopic quantities. For this reason, we investigate the averaged performance of our method by using the infinite-range model.

Suppose that the prior probability generates the original image in the infinite-range model like Eq. (1). Then the $i$ th pixel interacts all other pixels and the prior probability distribution is represented as

$$
P_{s}(\{\xi\})=\frac{1}{Z\left(\beta_{s}\right)} \exp \left[-\frac{\beta_{s}}{2 N} \sum_{i j}\left(\xi_{i}-\xi_{j}\right)^{2}\right],
$$

where $N$ is the system size and $Z\left(\beta_{s}\right)$ is the partition function of the ferromagnetic $Q$-Ising model. We assume that the original image is generated by this probability both in the bit-decomposed data case and the $Q$-Ising case. In this section, we adopt as the original image a snap shot of the system produced by the above probability.

We treat the Gaussian channel for simplicity. In our method, the Gaussian channel in which input data are expressed in a binary form is given by

$$
P_{k}\left(\left\{\tau_{i, k}\right\} \mid\left\{\xi_{i, k}\right\}\right)=\frac{1}{\sqrt{2 \pi} \tau} \exp \left[-\frac{1}{2 \tau^{2}} \sum_{i}\left(\tau_{i, k}-\tau_{0} \xi_{i, k}\right)^{2}\right],
$$

where $\tau_{i, k}$ is a Gaussian random variable with mean $\tau_{0} \xi_{i, k}$ and variance $\tau^{2}$. The input $\xi_{i, k}$ takes 0 or 1 . For comparison, the $Q$-Ising case is denoted

$$
P\left(\left\{\tau_{i}\right\} \mid\left\{\xi_{i}\right\}\right)=\frac{1}{\sqrt{2 \pi} \tau^{\prime}} \exp \left[-\frac{1}{2 \tau^{\prime 2}} \sum_{i}\left(\tau_{i}-\tau_{0}^{\prime} \xi_{i}\right)^{2}\right],
$$

where $\xi_{i}$ takes a value between 0 and $Q-1$, and $\tau_{i}$ is a Gaussian random variable with mean $\tau_{0}^{\prime} \xi_{i}$ and variance $\tau^{\prime 2}$. We calculate the posterior probability using Eqs. (16) and (17). In the bit-decomposed data case,

$$
\begin{aligned}
& P\left(\{\sigma\} \mid\left\{\tau_{1}\right\},\left\{\tau_{2}\right\}, \ldots,\left\{\tau_{Q-1}\right\}\right) \\
& \quad \sim \prod_{k}^{Q-1} P_{k}\left(\left\{\tau_{k}\right\} \mid\left\{\sigma_{k}\right\}\right) P_{m}(\{\sigma\}) \\
& \quad \sim \exp \left(-h \sum_{i} \sum_{k}\left(\sigma_{i, k}-\tau_{i, k}\right)^{2}-\frac{\beta_{m}}{2 N} \sum_{i, j}\left(\sigma_{i}-\sigma_{j}\right)^{2}\right) \\
& \quad \equiv \exp \left(-H_{\mathrm{eff}}\right) .
\end{aligned}
$$

In the $Q$-Ising case,

$$
P(\{\sigma\} \mid\{\tau\}) \sim \exp \left(-h \sum_{i}\left(\sigma_{i}-\tau_{i}\right)^{2}-\frac{\beta_{m}}{2 N} \sum_{i, j}\left(\sigma_{i}-\sigma_{j}\right)^{2}\right) .
$$

The difference of these expressions and Eqs. (13), (14) in the previous section is only that the coordination number $z$ became $N$.

We calculate the free energy from these probability distributions to clarify the behavior of macroscopic quantities. Since there is randomness in the field for the spin system described by the effective Hamiltonian $H_{\text {eff }}$ in Eqs. (18) and (19), the free energy must be averaged over the probability distribution of the random field in addition to the thermal average. Accordingly, we calculate the free energy per site by

$$
f=F / N=-\frac{1}{N \beta_{m}}[\ln Z] .
$$

By using the replica method, we obtain the following replicated partition function:

$$
\begin{aligned}
{\left[Z^{n}\right]=} & \sum_{\xi} \sum_{\tau} P_{s}(\{\xi\}) P(\{\tau\} \mid\{\xi\}) \operatorname{Tr}_{\sigma} e^{-H_{\mathrm{eff}}^{\mathrm{rep}}} \\
= & \operatorname{Tr}_{\xi} \int \prod_{i, k} d \tau_{i}^{(k)} \frac{1}{(\sqrt{2 \pi} \tau)^{(Q-1) N}} \\
& \times \exp \left[-\frac{1}{2 \tau^{2}} \sum_{i}\left(\tau_{i}-\tau_{0} \xi_{i}\right)^{2}\right] \\
& \times \exp \left[-\frac{\beta_{s}}{2 N} \sum_{i j}\left(\xi_{i}-\xi_{j}\right)^{2}\right] \operatorname{Tr}_{\sigma} \\
& \times \exp \left[-h \sum_{i} \sum_{k} \sum_{\alpha}\left(\tau_{i, k}-\sigma_{i, k}^{\alpha}\right)^{2}\right. \\
& \left.-\frac{\beta_{m}}{2 N} \sum_{i j} \sum_{\alpha}\left(\sigma_{i}^{\alpha}-\sigma_{j}^{\alpha}\right)^{2}\right]
\end{aligned}
$$

where $\alpha$ is the label of $n$ dummy replicas. Assuming replica symmetric ansatz $m_{\alpha}=m(\forall \alpha)$, the following expressions of the order parameters are derived: 


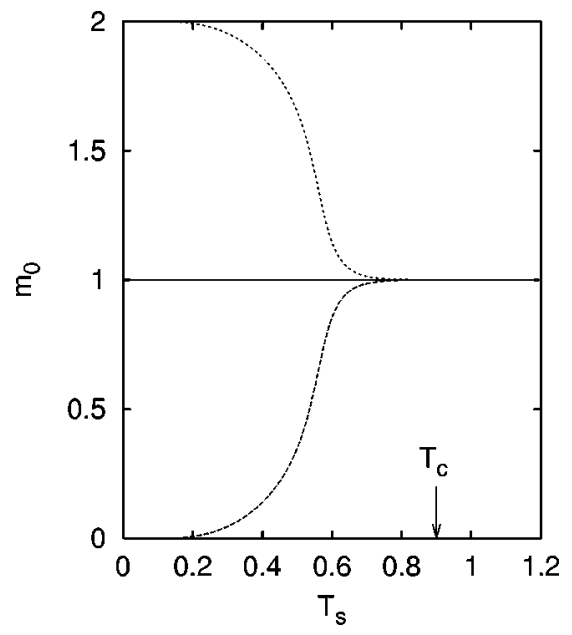

FIG. 2. Magnetization of the original image as a function of the source temperature for $Q=3$. The solid line corresponds to a globally stable solution $m_{0}=1$ and the dotted lines correspond to locally stable solutions $m_{0}=0$ and $m_{0}=1$.

$$
\begin{gathered}
{\left[\xi_{i}\right]=m_{0}=\frac{1}{Z\left(\beta_{s}\right)} \operatorname{tr}_{\xi} \xi e^{2 m_{0} \beta_{s} \xi-\beta_{s} \xi^{2}},} \\
{\left[\left\langle\sigma_{i}^{\alpha}\right\rangle\right]=m=\frac{1}{Z\left(\beta_{s}\right)} \operatorname{tr}_{\xi} e^{2 m_{0} \beta_{s} \xi-\beta_{s} \xi^{2}}} \\
\times \int D u_{1} \ldots, \int D u_{Q-1} \frac{\operatorname{tr}_{\sigma} \sigma \exp \left(\widetilde{H}_{\mathrm{eff}}\right)}{\operatorname{tr}_{\sigma} \exp \left(\widetilde{H}_{\mathrm{eff}}\right)}
\end{gathered}
$$

where we defined $D u \equiv(\mathrm{d} u / \sqrt{2 \pi}) e^{-u^{2} / 2}$ and

$$
\begin{aligned}
\widetilde{H}_{\mathrm{eff}} \equiv & 2 \beta_{m} m \sigma-\beta_{m} \sigma^{2}-h \sum_{k} \sigma_{k}^{2}+2 h \tau \sum_{k} u_{k} \sigma_{k} \\
& +2 h \tau_{0} \sum_{k} \xi_{k} \sigma_{k} .
\end{aligned}
$$

Equation (22) represents the magnetization of the original image in the infinite-range model. The behavior of the source magnetization $m_{0}$ as a function of the temperature $\beta_{s}$ is shown in Fig. 2. Equation (23) is the equation of state for the magnetization of the restored image. In the limit $N \rightarrow \infty$, the mean-square error [Eq. (10)] is rewritten as

$$
\begin{aligned}
H_{\mathrm{D}}= & \frac{1}{Z\left(\beta_{s}\right)} \operatorname{tr}_{\xi} e^{2 m_{0} \beta_{s} \xi-\beta_{s} \xi^{2}} \int D u_{1} \ldots, \int D u_{Q-1} \\
& \times\left\{\xi_{i}-\Omega\left(\left\langle\sigma_{i}^{\alpha}\right\rangle\right)\right\}^{2}
\end{aligned}
$$

We next assume a condition to compare the performance of restoration between using the bit-decomposed data and the $Q$-Ising form. The condition is that the distance between the original and the degraded images in our method is equal to that in the $Q$-Ising form. We may compare the difference of performance in disparate noisy channels by this condition. The distance between the original and degraded images in our method $H_{\mathrm{D}}^{\tau}(\mathrm{BDD})$ is expressed similarly to the above expression. It turns out that the distance $H_{\mathrm{D}}^{\tau}(\mathrm{BDD})$ becomes simple in the thermodynamic limit $N \rightarrow \infty$

$$
\begin{aligned}
H_{\mathrm{D}}^{\tau}(\mathrm{BDD})= & \frac{1}{N} \sum_{i}\left\{\left(\xi_{i, 1}+\xi_{i, 2}+\cdots+\xi_{i, Q-1}\right)\right. \\
& \left.-\left(\tau_{i, 1}+\tau_{i, 2}+\cdots+\tau_{i, Q-1}\right)\right\}^{2} \\
= & \left\langle\left(\sum_{k} \xi_{k}-\sum_{k} \tau_{k}\right)^{2}\right\rangle_{\tau_{1}, \ldots, \tau_{Q-1}, \xi_{1}, \ldots, \xi_{Q-1}} \\
= & (Q-1) \tau^{2}+\frac{\left(\tau_{0}-1\right)^{2}}{Z\left(\beta_{s}\right)} \\
& \times \operatorname{tr}_{\xi_{1}, \ldots, \xi_{Q-1}}\left[\sum_{k=1}^{Q-1} \xi_{k} e^{2 \beta_{s} m_{0}} \sum_{k} \xi_{k}-\beta_{s}\left(\sum_{k} \xi_{k}\right)^{2}\right] \\
= & (Q-1) \tau^{2} .
\end{aligned}
$$

When the mean $\tau_{0}$ of the Gaussian noise is one, the distance $H_{\mathrm{D}}^{\tau}$ (BDD) depends on the variance $\tau^{2}$ only. In the $Q$-Ising form, $H_{\mathrm{D}}^{\tau}(Q$ Ising $)$ is

$$
\begin{aligned}
H_{\mathrm{D}}^{\tau}(Q \text { Ising }) & =\frac{1}{N} \sum_{i}\left\{\xi_{i}-\tau_{i}\right\}^{2} \\
& =\tau^{\prime 2}+\frac{\left(\tau_{0}^{\prime}-1\right)^{2}}{Z\left(\beta_{s}\right)} \operatorname{tr}_{\xi}\left[\xi e^{2 \beta_{s} m_{0} \xi-\beta_{s} \xi^{2}}\right] \\
& =\tau^{\prime 2}
\end{aligned}
$$

where $\tau^{\prime 2}$ is the variance of a Gaussian channel in the $Q$-Ising form and is different from $\tau$ in the bit-decomposed data. When $\tau_{0}^{\prime}=0, H_{\mathrm{D}}^{\tau}(Q$ Ising $)=\tau^{\prime 2}$ similarly to the bitdecomposed data case. As the rate of degradation is equal to each other, namely $H_{\mathrm{D}}^{\tau}(\mathrm{BDD})=H_{\mathrm{D}}^{\tau}(Q$ Ising $)$, the variance of a noisy channel between in our method (26) and that in the $Q$-Ising form (27) satisfies the following relation:

$$
\tau=\frac{\tau^{\prime}}{\sqrt{Q-1}} .
$$

Next, we calculate the restoration of the gray-scale image when $Q=3$. We plot the magnetization $m_{0}$ of original image as a function of source temperature $T_{s}$ for $Q=3$ in Fig. 2. In the high-temperature region $T_{s} \rightarrow \infty$, the magnetization becomes $m_{0}=(0+1+2) / 3=1$, since each spin takes all the values with the same probability $1 / 3$. We see that two locally stable states are generated in the middle range of temperature. The globally stable state is the line of $m_{0}=1$. The transition temperature between the paramagnetic phase and the ferromagnetic phase is $T_{c} \sim 0.9$. These locally stable states become more stable with the decrease of temperature and correspond to the globally stable states $m_{0}=0$ and $m_{0}=2$ in $T_{s}=0$. That is, the system of the ferromagnetic $Q$-Ising model for $Q=3$ has triple degeneracy.

We use a snapshot of the system when the magnetization is $m_{0}=1$ at $T_{s}=0.75$ as the original image. When the distance between the original image and the degraded image is 

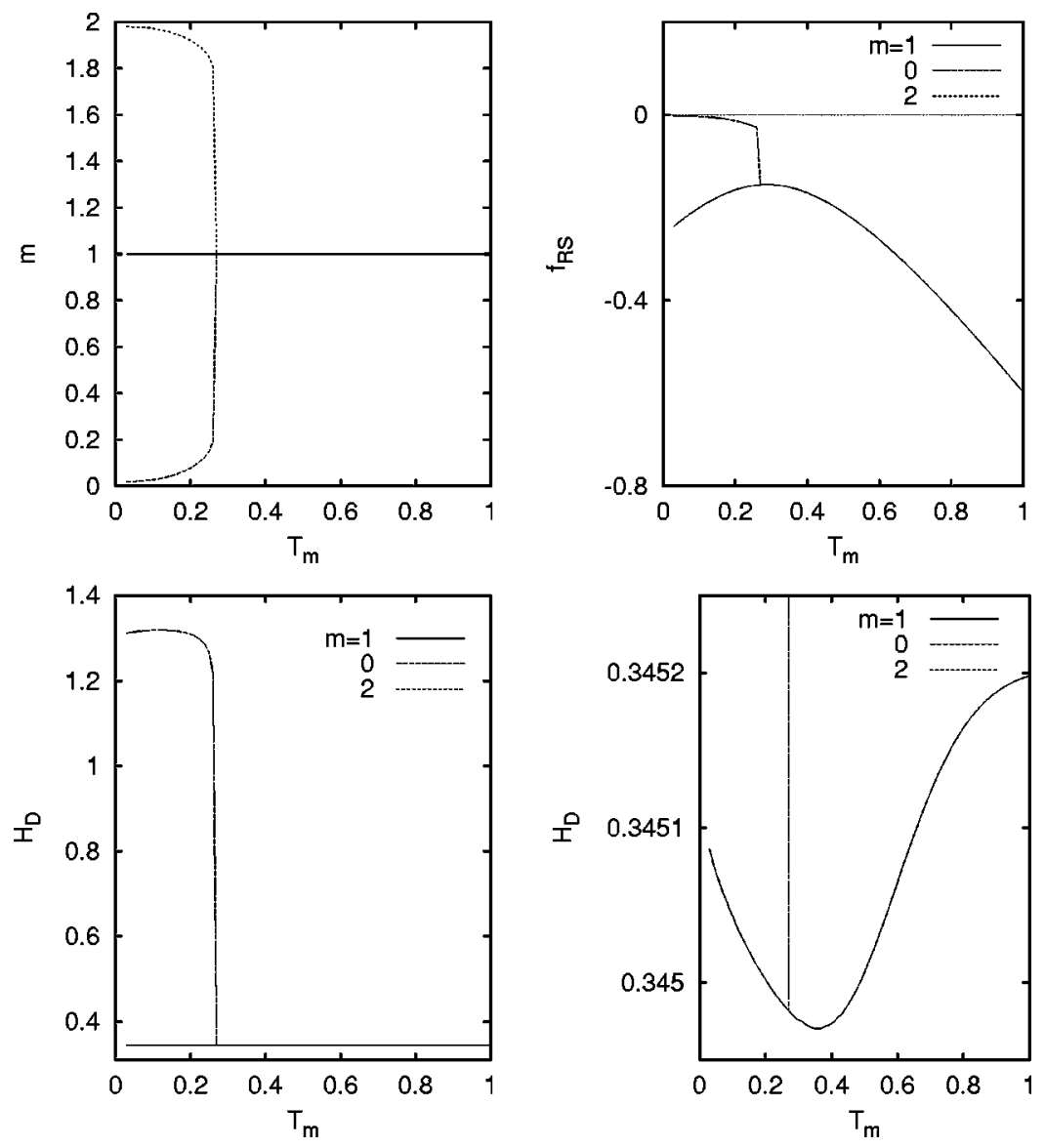

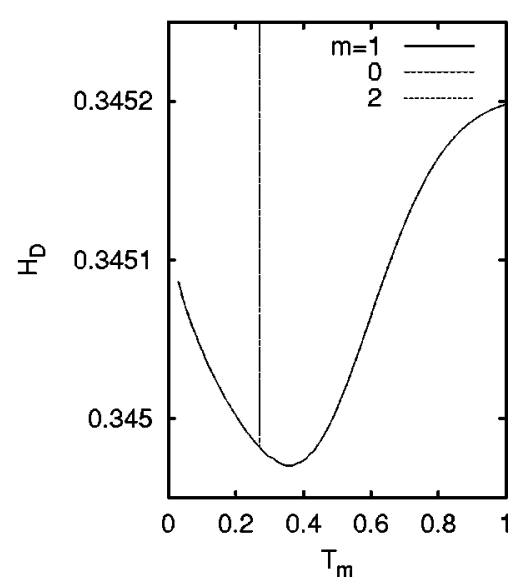

FIG. 3. The magnetization $m$ and corresponding free-energy $f_{\mathrm{RS}}$ are plotted in the upper-left and upper-right figures. The mean-square error is plotted in the lower-left figure. The lower-right figure represents an enhancement of the meansquare error around the optimal values. The solid line corresponds to the globally stable solution.
$H_{\mathrm{D}}^{\tau}=1.0$, macroscopic quantities behave as in Fig. 3. The magnetization $m$ of Eq. (23) at the ratio of hyperparameters $H \equiv h / \beta_{m}=0.25$ has three stable states at $T_{m}=0$ as $m_{0}$ we see in the original image. At low temperature, the system has one globally stable state $m=1$ and two locally stable states $m=0$ and 2 . However, these locally stable states vanish as the ratio of hyperparameters $H$ becomes large. Seeing the free energy in Fig. 3, we find that the state of system branches out into a global minimum and local minima at $T_{m}=0.27$.

The mean-square error $H_{\mathrm{D}}$ using $m$ of the global minimum is smaller than that using $m$ of local minimum in all temperature regions and gives the optimal value for the ratio of hyperparameters $H$. The mean-square error $H_{\mathrm{D}}$ between the $m$ of global minimum and the original image $m_{0}$ in some ratio of hyperparameters $H$ are described in Fig. 4. The smallest mean-square error is given at $T_{m}=0.75$ and $H$ $=0.75$. When the distance between the original and degraded images is $1.0\left(H_{\mathrm{D}}^{\tau}=1.0\right)$, we see that the deviation $\tau$ of Gaussian noise becomes $1 / \sqrt{Q-1}=1 / \sqrt{2}$ by Eq. (26). Therefore, when the ratio of hyperparameters corresponds to that of the prior parameters $\left[H=h / \beta_{m}=\left(\tau_{0} / 2 \tau^{2}\right) / \beta_{s}\right.$ $=0.75]$, the optimal restoration is given at the temperature corresponding to the source temperature $\left(T_{m}=T_{s}=0.75\right)$ similarly to the Ising model. At high temperatures $T_{m} \rightarrow \infty$, all states appear with equal probability. Then the thermal
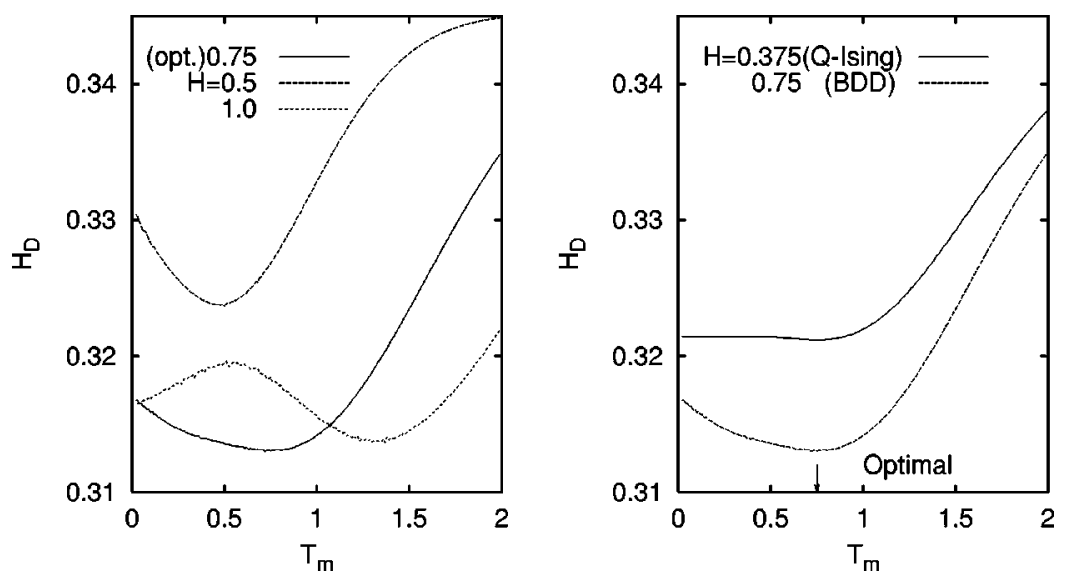

FIG. 4. Left figure is the mean-square error as a function of temperature for several $H$. The solid line corresponds to the optimal value, $T_{m}=T_{s}$. Right figure represents a comparison between the mean-square error in the bit-decomposed data and the $Q$-Ising form for the optimal hyperparameters $H=H^{\text {opt }}$. The solid line is that for the BDD case and the dotted line is the $Q$-Ising case. 

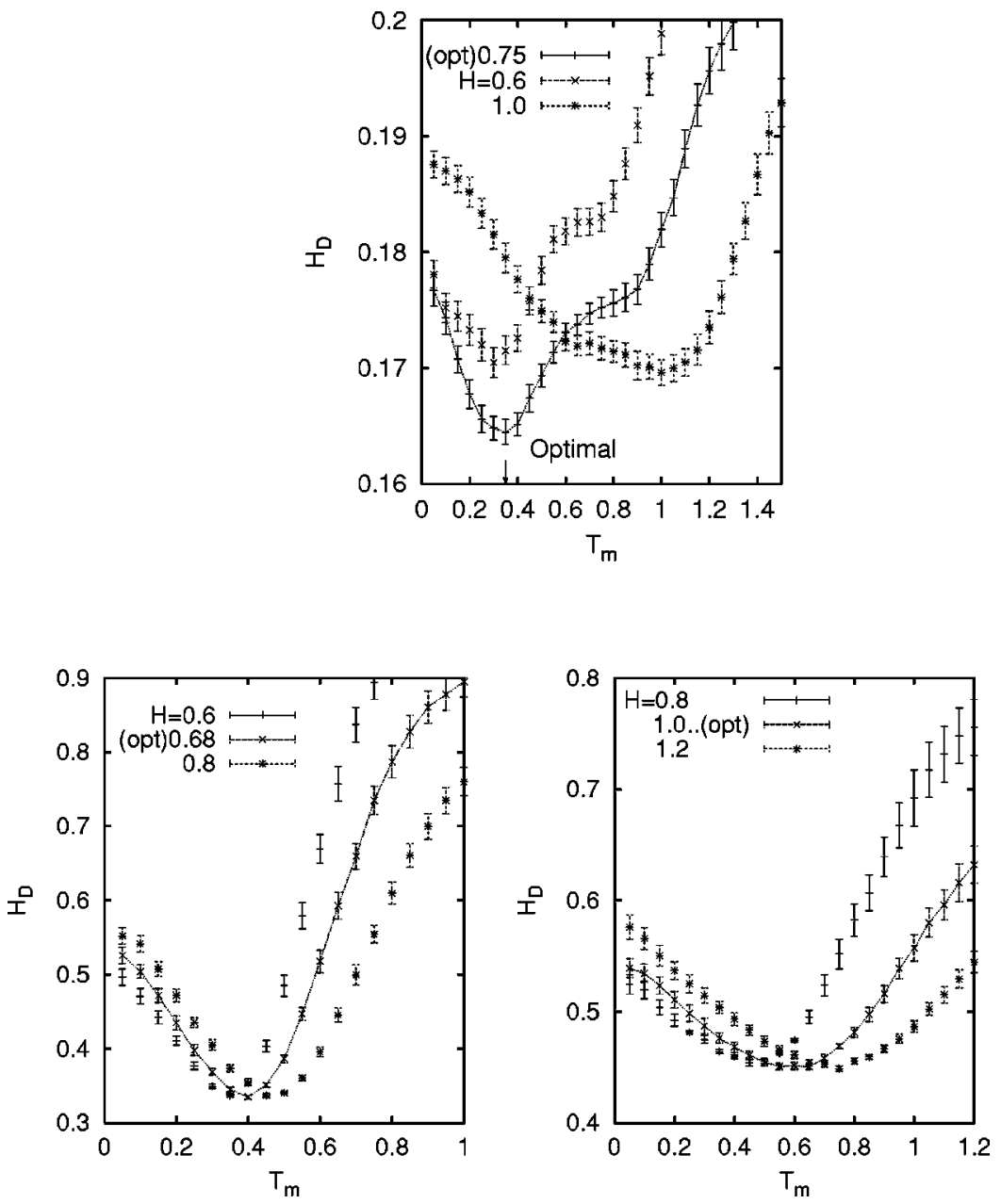

average of a spin $\sigma$ becomes $\langle\sigma\rangle=(0+1+2) / 3=1$ at all pixels. The mean square error is

$$
H_{\mathrm{D}}\left(T_{m} \rightarrow \infty\right)=\frac{\sum_{\xi=0}^{3}(\xi-1)^{2} e^{2 m_{0} \beta_{s} \xi-\beta_{s} \xi^{2}}}{\sum_{\xi=0}^{3} e^{2 m_{0} \beta_{s} \xi-\beta_{s} \xi^{2}}} \sim 0.3452
$$

If the distance between the original and degraded images, $H_{\mathrm{D}}^{\tau}(\mathrm{BDD})$ or $H_{\mathrm{D}}^{\tau}(Q$ Ising $)$, is extremely large, the performance of restoration does not become smaller than this value.

We compare the optimal performance of restoration using our method with that using the $Q$-Ising form under the condition that the degradation of image in our method is equivalent to the one in the $Q$-Ising form, namely, $H_{\mathrm{D}}^{\tau}(\mathrm{BDD})$ $=H_{\mathrm{D}}^{\tau}$ ( $Q$-Ising form). In the $Q$-Ising form case, the deviation is $\tau=1.0$ by Eq. (27) when $H_{\mathrm{D}}^{\tau}=1.0$. The optimal restoration in the $Q$-Ising form is also given in the ratio of hyperparameters corresponding to that of source parameters $\left[H=h / \beta_{m}\right.$ $\left.=\left(\tau_{0} / 2 \tau^{2}\right) / \beta_{m}=0.375\right]$. The optimal performance of restoration in both our method and the $Q$-Ising form is shown in Fig. 4. We see that the restoration by our method gives a better performance than that of the $Q$-Ising form. data point.
FIG. 5. The mean-square error in the $Q=4$ case is calculated by Monte Carlo simulation (the upper figure). Averages over ten samples that (system size is $100 \times 100$ ) are taken at each data point. The ratio of hyperparameters $H$ is chosen to be $H=0.6,0.75$ (optimal), and 1.0. The meansquare error when $Q=8, p=0.15, H_{\mathrm{D}}^{\tau} \sim 1.0$ (the lower figures). The lower-left figure represents the mean-square error in $T_{s}=0.4$, and the ratio of hyperparameters $H$ is chosen to be $H$ $=0.6,0.68$ (optimal), and 0.8. The lower-right figure represents the mean-square error in $T_{s}$ $=0.6$, and the ratio of hyperparameters $H$ is chosen to be $H=0.8,1.0$ (optimal), and 1.2.

\section{MONTE CARLO SIMULATION}

In this section, we investigate realistic pictures in two dimensions and check the results obtained in the infiniterange model. It is, however, difficult to investigate the restoration of real images by analytical methods. We discuss the restoration of two-dimensional images by means of Monte Carlo simulations.

We suppose that the original image is generated by the Boltzmann probability distribution as we did in the infiniterange model case and we use a snap shot of the $Q$-Ising model at a temperature as the original image.

In digital images, the pixel takes discrete values. Even if real-valued Gaussian noise is added in the degradation process, we have to regard such a noise as the discrete noise (for example, binary noise) when both the original and the degraded images are digital. (This is essentially the same situation as in a error-correcting codes [11]). Therefore, we consider the binary noise caused by the binary symmetric channel with the error probabilities $p=0.10(Q=4)$ and $p$ $=0.15(Q=8)$, which correspond to the parameters $\beta_{\tau}$ $\sim 2.2$ and $\beta_{\tau} \sim 1.7$ by Eq. (5). The size of digital image is $100 \times 100$ and averages over ten samples are taken at each

Figure 5 (the upper figure) represents the mean-square error of several $H$ when $Q=4, T_{s}=0.35, m_{0} \sim 1.2, p$ 
$=0.10$, and $H_{\mathrm{D}}^{\tau} \sim 0.30$. The optimal restoration is given at $T_{m}=T_{s}, H=h / \beta_{m}=\beta_{\tau} / \beta_{s} \sim 0.75$, similar to in the infiniterange model.

The mean square errors for $Q=8, T_{s}=0.4,(0.6), m_{0}$ $\sim 2.2,(3.0), p=0.15$, and $H_{\mathrm{D}}^{\tau} \sim 1.0$ are also shown in Fig. 5 (the lower two figures.). We see that the best performance of restoration at each temperature is obtained at the ratio of hyperparameters $H$ corresponding to that of the prior parameters, and the performance of restoration in $T_{s}=0.4$ (the lower left) is better than that in $T_{s}=0.6$ (the lower right) as written before.

\section{MEAN-FIELD ANNEALING}

The amount of computation required for restoration by means of Monte Carlo simulation is enormous. Hence, in the present section, we reconstruct the original image from binary degraded images by means of the mean-field annealing $[12,13]$ with periodic boundary conditions. This method enables us to search for the optimal solution quickly. We apply the mean-field approximation to the posterior probability distribution

$$
\begin{aligned}
P\left(\{\boldsymbol{\sigma}\} \mid\left\{\boldsymbol{\tau}_{\mathbf{1}}\right\},\left\{\boldsymbol{\tau}_{\mathbf{2}}\right\}, \ldots,\left\{\boldsymbol{\tau}_{\mathbf{Q}-\mathbf{1}}\right\}\right) & =\frac{1}{Z\left(\beta_{m}, h\right)} \exp \left(-h \sum_{i} \sum_{k}\left(\sigma_{i, k}-\tau_{i, k}\right)^{2}\right. \\
& \left.-\frac{\beta_{m}}{2 N} \sum_{i, j}\left(\sigma_{i}-\sigma_{j}\right)^{2}\right) .
\end{aligned}
$$

In order to treat each site separately, we trace out with respect to all pixels besides the $i$ th pixel in the above probability distribution

$$
\begin{aligned}
\rho_{i j}(n)= & \prod_{k l \neq i j} \operatorname{tr}_{\sigma_{1}, \ldots, \sigma_{Q-1}} P\left(\{\boldsymbol{\sigma}\} \mid\left\{\boldsymbol{\tau}_{\mathbf{1}}\right\},\left\{\boldsymbol{\tau}_{\mathbf{2}}\right\}, \ldots,\left\{\boldsymbol{\tau}_{\mathbf{Q}-\mathbf{1}}\right\}\right) \\
= & \operatorname{Tr}_{\sigma_{1}, \ldots, \sigma_{Q-1}} P\left(\{\boldsymbol{\sigma}\} \mid\left\{\boldsymbol{\tau}_{\mathbf{1}}\right\},\left\{\boldsymbol{\tau}_{2}\right\}, \ldots,\left\{\boldsymbol{\tau}_{\mathbf{Q}-\mathbf{1}}\right\}\right) \\
& \times \delta\left(n, \sigma_{i j}\right),
\end{aligned}
$$

where $(i j)$ represents the site index in two dimensions: $i$ and $j$ are the coordinates of $x$ and $y$ axes, respectively. This expression is called the marginal probability distribution. According to mean-field approximation, the posterior probability distribution (30) is approximated by the product of the marginal probability distribution (31) as follows

$$
P\left(\{\boldsymbol{\sigma}\} \mid\left\{\boldsymbol{\tau}_{\mathbf{1}}\right\},\left\{\boldsymbol{\tau}_{\mathbf{2}}\right\}, \ldots,\left\{\boldsymbol{\tau}_{\mathbf{Q}-\mathbf{1}}\right\}\right) \simeq \prod_{(i j)} \rho_{i j}(n) .
$$

The recursion relation for the iterative algorithm is derived from a variational principle. To derive the recursion relation, we substitute the expression of approximation (32) to the free energy

$$
F(\rho)=E(\rho)-T_{m} S(\rho) .
$$

TABLE I. Comparison of performance between our method and the $Q$-Ising form. The ratios of pixels at which the nearestneighboring pixels take the same value in the original image, the degraded image and restored image are represented as "NNP1."

\begin{tabular}{lcccc}
\hline \hline Original Image & $H_{D}^{\text {opt. }}$ & NNP1-O & NNP1-D & NNP1-R \\
\hline Chair (BDD) & 0.10467500 & 0.827925 & 0.023925 & 0.870800 \\
Chair $(Q$-Ising) & 0.13967500 & 0.827925 & 0.127746 & 0.849700 \\
Girl (BDD) & 0.22960000 & 0.552725 & 0.019000 & 0.636575 \\
Girl $(Q$-Ising) & 0.26657500 & 0.552725 & 0.092100 & 0.452850 \\
House (BDD) & 0.38037500 & 0.538375 & 0.018000 & 0.374400 \\
House $(Q$-Ising) & 0.36840000 & 0.538375 & 0.079225 & 0.469675 \\
Lena (BDD) & 0.35317500 & 0.450500 & 0.016575 & 0.467425 \\
Lena $(Q$-Ising) & 0.45800000 & 0.450500 & 0.056086 & 0.449450 \\
Mandrill (BDD) & 0.64400000 & 0.147900 & 0.008950 & 0.136975 \\
Mandrill $(Q$-Ising) & 0.55120000 & 0.147900 & 0.021750 & 0.111100 \\
\hline
\end{tabular}

The variation of the above free energy is calculated with respect to the marginal probability distribution at a certain site: $\rho_{i j}$. Finally, the recursion relation with respect to the local magnetization for the iteration algorithm is obtained as

$$
\begin{gathered}
m_{i j}^{t+1}=\frac{\sum_{\sigma_{i j}=0}^{Q-1} \sigma_{i j} \exp \left(H_{\mathrm{MF}}^{t}\right)}{Z_{\mathrm{MF}}}, \\
H_{\mathrm{MF}}^{t}=\frac{\beta_{m}}{2}\left(m_{i, j+1}^{t}+m_{i, j-1}^{t}+m_{i+1, j}^{t}+m_{i-1, j}^{t}\right) \sigma_{i j}-\beta_{m} \sigma_{i j}^{2} \\
-h \sum_{k}\left(\sigma_{i j,(k)}\right)^{2}+2 h \sum_{k} \tau_{i j,(k)} \sigma_{i j,(k)},
\end{gathered}
$$

where $Z_{\mathrm{MF}}$ is the normalization constant in the mean-field approximation. We solve the above relations numerically under the convergence condition

$$
\epsilon^{(t)} \equiv \frac{1}{N} \sum_{(i j)}^{N}\left|m_{i j}^{(t+1)}-m_{i j}^{(t)}\right|<10^{-8},
$$

and obtain approximately the restored image at each temperature. The annealing schedule is set at $\Delta T_{m}=0.01$.

We compare the performance of restoration using our method with that using the $Q$-Ising form for five kinds of standard images [14]. We choose $Q=8$, system size $=200$ $\times 200$, and $H_{\mathrm{D}}^{\tau} \simeq 1.00$. The result is shown in Table I. The original versions of these five standard image are shown in Fig. 6.

The original, degraded, and optimal restored images in our method and the $Q$-Ising form are shown in Fig. 7 for the case of "lena."

In Table I, the ratio of pixels at which the nearestneighboring pixels take the same value is also represented. We see that the performance of restoration using our method is better than that using the $Q$-Ising form in the standard pictures "chair" and "girl." However, the $Q$-Ising form is better in "house," "lena," and "mandrill." One of the differences is that "chair" and "girl" have a lot of large parts 


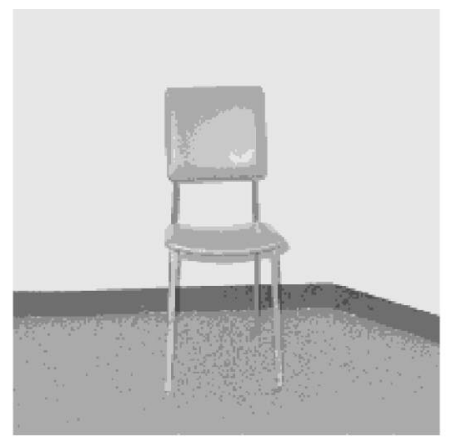

"chair"
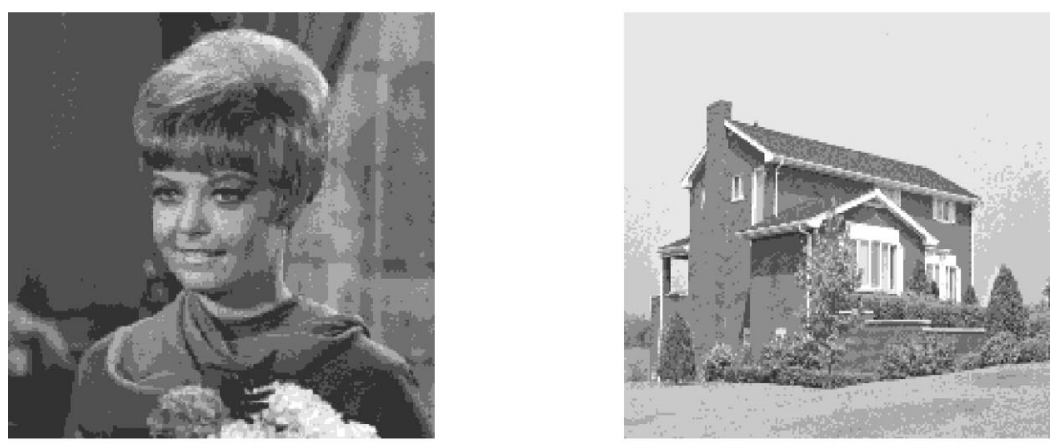

"girl" (left) and "house" (right)
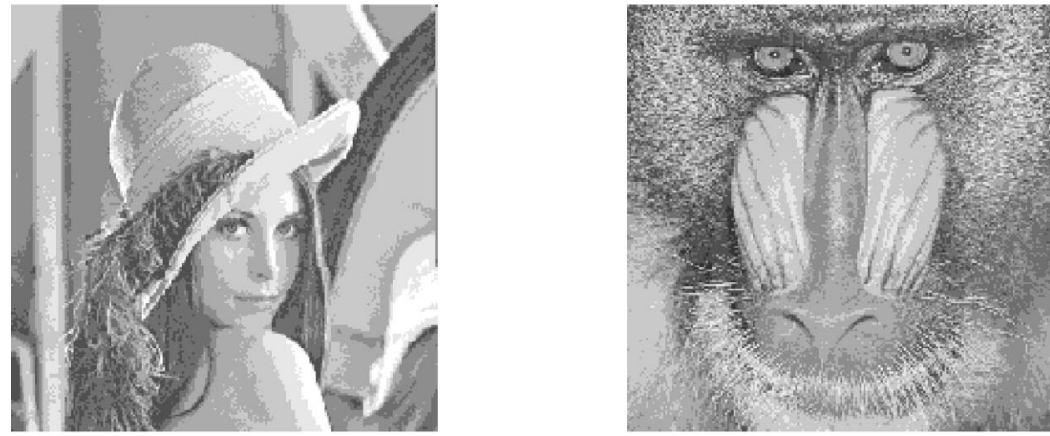

"lena" (left) and "mandrill" (right)
FIG. 6. Standard images: "chair," "girl," "house," "lena," and "mandrill." and do not have short edges compared with the other pictures as we see intuitively. In Table I, the "chair" that has obviously large parts is quantitatively expressed by the ratio of pixels at which the nearest-neighboring pixels take the same value. However, the ratio in "girl" is as much as that in "house" and "lena."

In order to investigate this aspect in more detail, we show the ratio of pixels at which the difference among the value of the nearest-neighboring pixels is smaller than two in Table II. As seen in Table II, "girl" is similar to "chair" rather than "house" and "lena." The effects of noise in the bitdecomposed data and the $Q$-Ising form are clarified by comparing the NNP1-D in Table I and NNP2-D in Table II. NNP1-D in our method is smaller than that in the $Q$-Ising form for all kinds of standard images, but the decrease of NNP2 from the original image to the degraded image in our method is small compared with that in the $Q$-Ising form for five standard images. That is, the noise in our method affects the original image widely, but the shift of value in a site is small. On the other hand, the shift of value in a site is large in the $Q$-Ising form. Therefore, the noise in our method has the effect of smoothing, and the restoration using our method is efficient for original images in which eliminating noise is more significant rather than preserving informations of original image as "chair" and "girl."

Furthermore, we compare the performance using our method and in the $Q$-Ising form for the $H_{\mathrm{D}}^{\tau} \sim 2.0$ case. Other conditions are the same as the previous case. We may simply investigate the tolerance of our method and the $Q$-Ising form against noise by comparing $H_{\mathrm{D}}^{\tau} \sim 1.0$ and 2.0 cases. The performance of restoration is shown in Table III and the resultant image is presented in Fig. 8 for the case of "lena." The performance of restoration is clearly worse than that in the previous case for all pictures, and the difference of performance between our method and the $Q$-Ising form is clear. Moreover, the restoration using our method gives a better performance than that using the $Q$-Ising form in the standard image "house." The result is different from that when $H_{\mathrm{D}}^{\tau}$ $\sim 1$.0. In the $Q$-Ising form, large clusters remain in the re- 


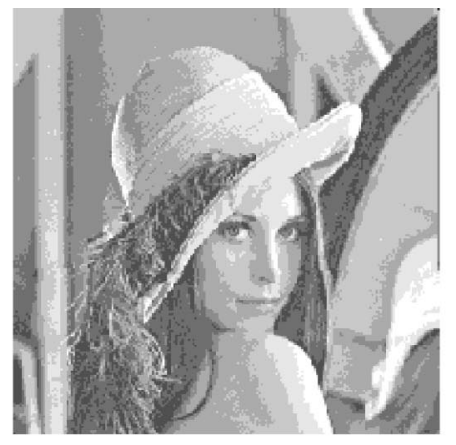

Original image
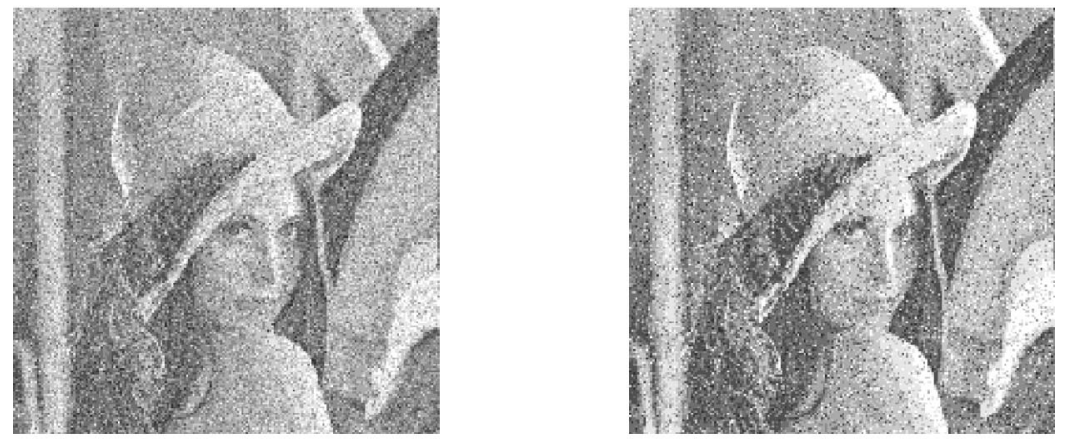

Degraded images
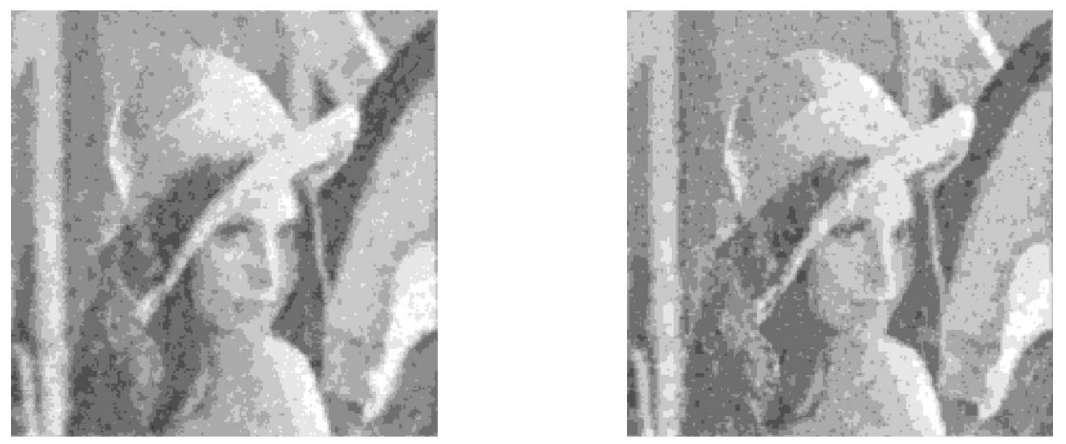

Restored images

TABLE II. Ratio of pixel at which the difference among the value of the nearest-neighboring pixels is smaller than two in the original, degraded, and restored images.

\begin{tabular}{lcccc}
\hline \hline Original Image & NNP2-O & NNP2-D & NNP2-R & $H_{D}^{\text {opt. }}$ \\
\hline Chair (BDD) & 0.955475 & 0.522025 & 0.998600 & 0.10467500 \\
Chair ( $Q$-Ising) & 0.955475 & 0.476000 & 0.999750 & 0.13967500 \\
Girl (BDD) & 0.945025 & 0.450400 & 0.993850 & 0.22960000 \\
Girl $(Q$-Ising) & 0.945025 & 0.438650 & 0.979750 & 0.26657500 \\
House (BDD) & 0.838400 & 0.442450 & 0.959600 & 0.38037500 \\
House $(Q$-Ising) & 0.838400 & 0.430775 & 0.967225 & 0.36840000 \\
Lena (BDD) & 0.879325 & 0.413950 & 0.989825 & 0.35317500 \\
Lena ( $Q$-Ising) & 0.879325 & 0.402920 & 0.981625 & 0.34800000 \\
Mandrill (BDD) & 0.615325 & 0.298100 & 0.934050 & 0.64400000 \\
Mandrill $(Q$-Ising) & 0.615325 & 0.272400 & 0.801025 & 0.55120000 \\
\hline \hline
\end{tabular}

FIG. 7. Standard image "lena." Left-side figures represent the restoration using the bitdecomposed data, and right-side figures represent the restoration using the $Q$-Ising form for $H_{\mathrm{D}}^{\tau}$ $\sim 1.0$.
TABLE III. Comparison of performance between using our method and the $Q$-Ising form for five standard images when $H_{\mathrm{D}}^{\tau}$ $\sim 2$.0. In this case, the restoration using our method is more efficient than that using the $Q$-Ising form in three standard images: "chair," "girl," and "house."

\begin{tabular}{lcccc}
\hline \hline Original Image & $H_{D}^{\text {opt. }}$ & NNP-O & NNP-D & NNP-R \\
\hline Chair (BDD) & 0.15512500 & 0.827925 & 0.006525 & 0.873225 \\
Chair ( $Q$-Ising) & 0.34862500 & 0.827925 & 0.050550 & 0.629175 \\
Girl (BDD) & 0.34907500 & 0.552725 & 0.007175 & 0.653800 \\
Girl $(Q$-Ising) & 0.51250000 & 0.552725 & 0.044300 & 0.519775 \\
House (BDD) & 0.53577500 & 0.538375 & 0.006625 & 0.485500 \\
House $(Q$-Ising) & 0.62777500 & 0.538375 & 0.034900 & 0.276275 \\
Lena (BDD) & 0.54720000 & 0.450500 & 0.005400 & 0.507025 \\
Lena $(Q$-Ising) & 0.48840000 & 0.450500 & 0.024625 & 0.543100 \\
Mandrill (BDD) & 0.89227500 & 0.147900 & 0.004225 & 0.469375 \\
Mandrill $(Q$-Ising) & 0.76495000 & 0.147900 & 0.008175 & 0.254500 \\
\hline
\end{tabular}




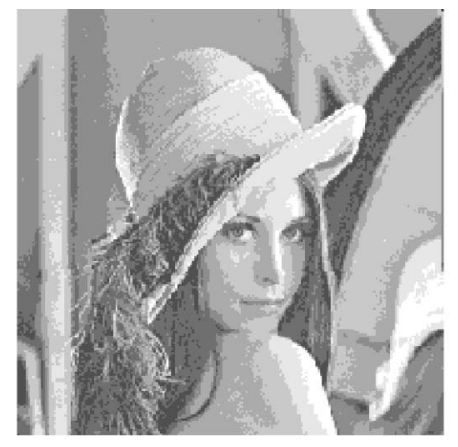

Original image
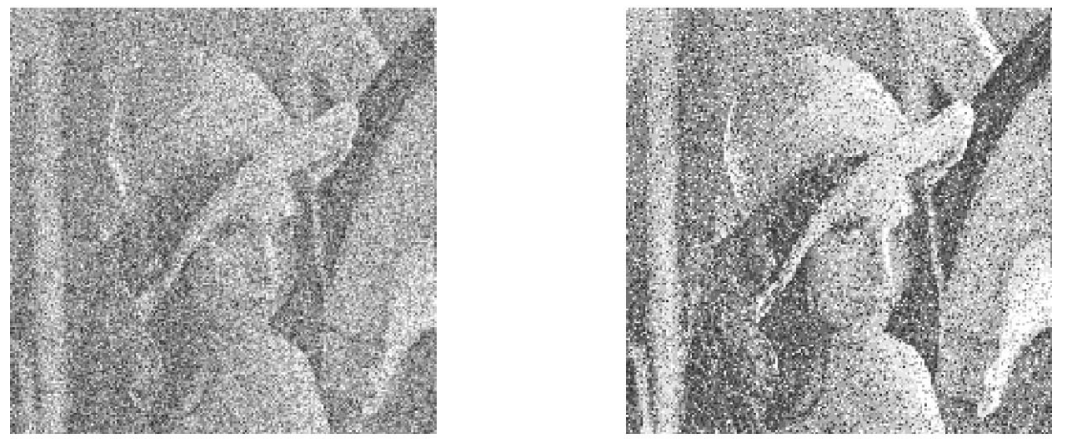

Degraded images
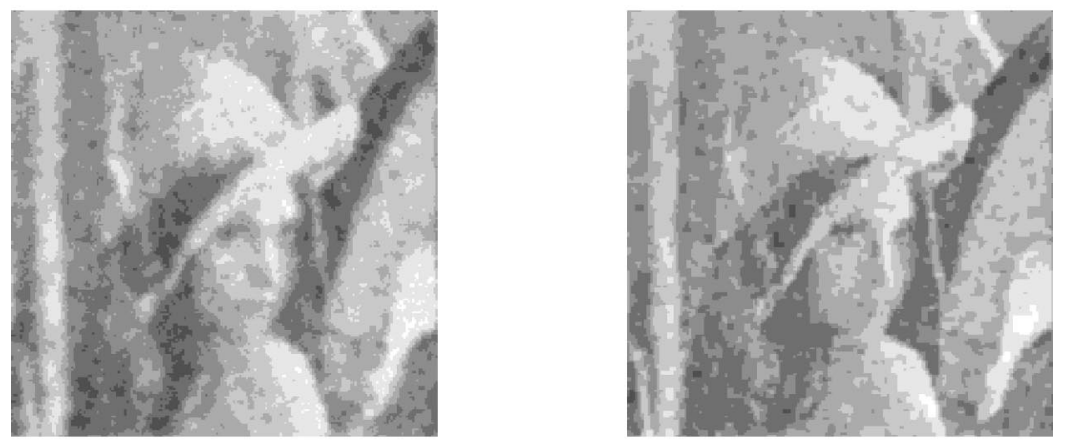

Restored images

stored image as in Fig. 8. Accordingly, we conclude that the restoration using the bit-decomposed data is not affected by the noise compared with that using the $Q$-Ising form in this case.

Consequently, we found that the restoration using our method is more efficient than that in the $Q$-Ising form in original images where the nearest-neighboring pixels take the close values. The reason is that to eliminate the added noise is more significant than the information of the original image in such images. Regardless of the $Q$-Ising form and our method, the restoration of such images tend to give good performance compared with that of other images in the image restoration using the method of statistical mechanics. Moreover, our method may give the adequate restored image in the original images "chair," "girl," and "house" even if the noise is strong to a certain extent: for example, $H_{\mathrm{D}}^{\tau}$ $\sim 2.0$.

Finally, we try to restore the original image by using the composition of our method and the $Q$-Ising form. Two processes are considered in restoration using the composition.
FIG. 8. Standard image "lena." Left-side figures represent the restoration using the bitdecomposed data, and right-side figures represent the restoration using the $Q$-Ising form for $H_{\mathrm{D}}^{\tau}$ $\sim 2.0$.
One is the method of restoration where the original image is restored from $Q-1$ sets of binary degraded images, which are generated from the received, degraded $Q$-value image by the method of threshold division. Another is the method of restoration where the original image is restored by using the $Q$-Ising form after translating $Q-1$ sets of binary degraded images into a $Q$-value degraded image. These restoration processes are shown in Fig. 9.

The second process is not efficient in the restoration of the gray-scale image because the performance of restoration is worse than that both using our method and the $Q$-Ising form. On the other hand, the first process gives interesting results as in Table IV when $H_{\mathrm{D}}^{\tau} \sim 1.0$. Noting Table IV, we find that this method gives a better performance than our method and the $Q$-Ising form for all standard images: "chair," "girl," "house," "lena," and "mandrill." The restoration of standard images using this method for the case of "lena" is shown in Fig. 10. This idea that, after a $Q$-value data are received, they are decomposed into binary data has been already used in the field of engineering. The filter is called the 


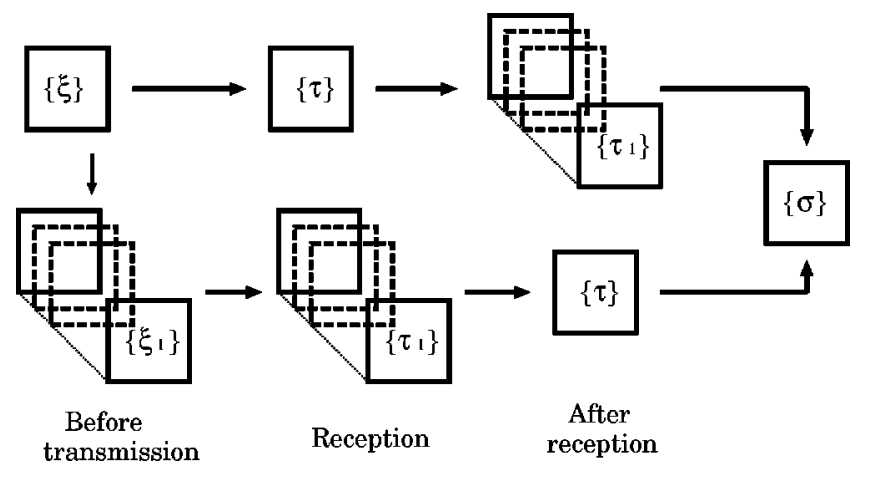

FIG. 9. Restoration processes using the composition of both our method and the $Q$-Ising form. In the upper process (first process), we decompose a $Q$-value degraded image into $Q-1$ sets of binary degraded images after we received a $Q$-value degraded image. Using the binary degraded images, we restore the original image. On the other hand, in the lower process (second process), we received $Q-1$ sets of binary degraded images. After they are translated into a $Q$-value degraded image, we restore the original image using the $Q$-Ising form.

Stack filter [15] which works on decomposed binary data after reception.

The Stack filter, by means of the method of statistical mechanics as the first process, gives a better performance than the other two methods, but the theoretical framework is not clear. Because we have to assume the noisy process matching the degraded data in the Bayesian viewpoint, it is very difficult for us to estimate the noisy channel matching such binary data. Accordingly, it is very well possible that the hyperparameter $h$ cannot be estimated appropriately for the hyperparameters estimation in the Stack filter by means of the method of statistical mechanics.

\section{SUMMARY AND DISCUSSIONS}

In this paper, we investigated the restoration of the grayscale image using the bit-decomposed data instead of the conventional $Q$-Ising form and found the conditions to show that our method is more efficient than the method of the $Q$-Ising form.

In the infinite-range model, we analyzed the image restoration when the original image is affected by the Gaussian noise, and obtained the static properties of image restoration.

TABLE IV. Comparison of performance among using our method, the $Q$-Ising form, and the composition for five standard images when $H_{\mathrm{D}}^{\tau} \sim 1.0$. In this case, the restoration using the composition is more efficient than that using our method and the $Q$-Ising form in all standard images.

\begin{tabular}{lccc}
\hline \hline Original Image & Composition & BDD & $Q$-Ising \\
\hline Chair & 0.09427500 & 0.10467500 & 0.13967500 \\
Girl & 0.18190000 & 0.22960000 & 0.26657500 \\
House & 0.32717500 & 0.38037500 & 0.36840000 \\
Lena & 0.25742500 & 0.35317500 & 0.34800000 \\
Mandrill & 0.52147500 & 0.64400000 & 0.55120000 \\
\hline \hline
\end{tabular}
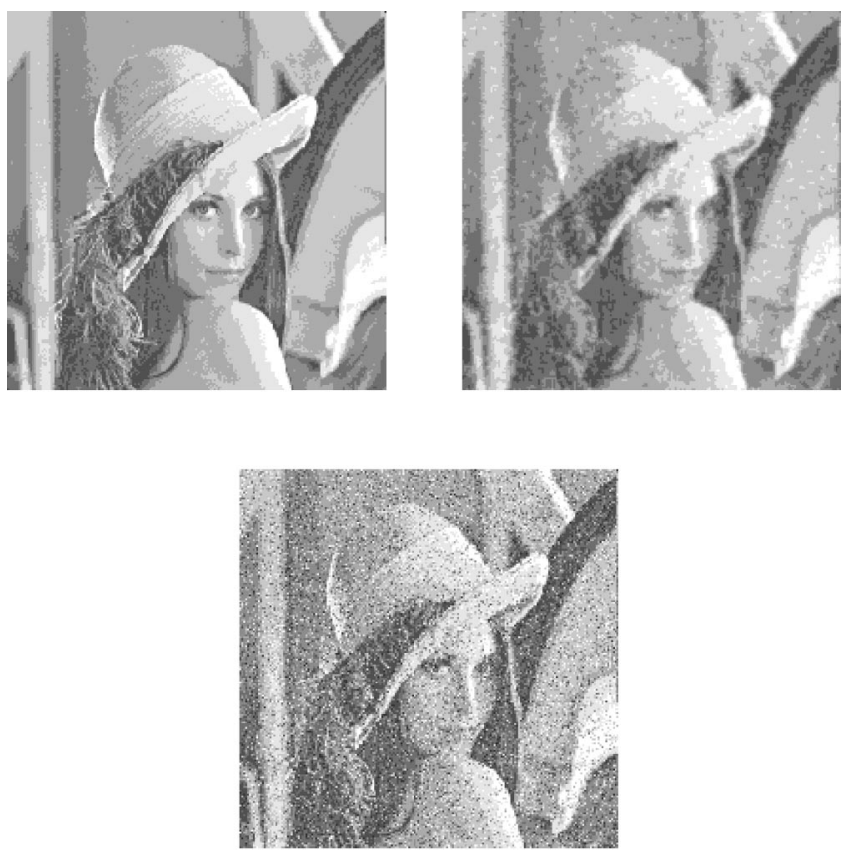

FIG. 10. Standard image "lena." The left, center, and right figures are the original, degraded, and restored images, respectively, in the composition process when $H_{\mathrm{D}}^{\tau} \sim 1.0$.

In order to obtain the averaged performance, we calculated the restoration of the gray-scale image by means of the replica method. Then we found that the mean-square error has a minimum at the finite temperature for any ratio of the hyperparameters $H$. However, when the noise rate is extremely large, the minimum sinks under the mean-square error $H_{\mathrm{D}}\left(T_{m} \rightarrow \infty\right)$ in the high-temperature limit. In other words, we may only obtain the restored image in which all states appear with equal probability as the optimal restored image when the original image is very corrupted. The best performance of restoration is given at the temperature at which the original image is generated, when the ratio of hyperparameters corresponds to that of the prior parameters. This result is also common between the Ising spin and the $Q$-Ising spin cases. Furthermore, we obtained the quantitative result that the performance of restoration using our method is better than that using the $Q$-Ising form when $H_{\mathrm{D}}^{\tau}(\mathrm{BDD})$ $=H_{\mathrm{D}}^{\tau}(Q$ Ising $)$.

We analyzed the restoration of realistic images in two dimensions by means of the Monte Carlo method and the mean-field annealing approximately. Using the Monte Carlo method, we confirmed the result of the infinite-range model that the mean-square error gives the optimal minimum value at the temperature corresponding to the source temperature when the ratio of hyperparameters corresponds to prior parameters for a snapshot of the $Q$-Ising model for $Q=4$ and 8 . We restored five standard images by means of the mean-field annealing and found that the restoration using our method is better than that using the $Q$-Ising form in the original images, where keeping the information of original image is not significant compared with eliminating the added noise as "chair" and "girl." The reason is that the effects of noise in our method contain the effect of smoothing. However, our 
method is not efficient in images that need information of the original image strongly.

Thus, there are simply two types in natural images. One is the image that consists of a little long edges and large surfaces, and those images depend on a basic and common property of natural image strongly. Another is the image that has many short edges and small clusters, and it is difficult to distinguish whether the original or degraded images in such images. Therefore, not only corrupted parts but correct parts may be destroyed by the effect of smoothing in such images. In restoration of images by means of the method of statistical mechanics, the effect of smoothing is strong compared with keeping the information of the original image that the degraded image contains because we use it as common property of natural images. Therefore, we need some additional information on the original image.

Furthermore, we found also that the restoration using our method is not affected by noise compared with that using the $Q$-Ising form. One of the reasons is due to the output, namely the degraded data, which takes the close value to input data by the effects of noise in our method. This is the most significant property of our method. We expect that the restoration using our method gives a further better performance when the $Q$ value is large. Because we could not distinguish visually the difference between close values with increasing the gray-scale level ( $Q$ value), the effect of noise may be suppressed visually. Accordingly, we may be able to obtain the restored image that looks closer to the original image intuitively.

\section{ACKNOWLEDGMENT}

The authors thank Professor Hidetoshi Nishimori for useful discussions.
[1] H. Nishimori, Statistical Physics of Spin Glass and Information Theory: An Introduction (Oxford University Press, New York, 2001).

[2] S. Geman and D. Geman, IEEE Trans. Pattern Anal. Mach. Intell. 6, 721 (1984).

[3] D. Geiger and F. Girosi, IEEE Trans. Pattern Anal. Mach. Intell. 15, 401 (1991).

[4] J. M. Pryce and A. D. Bruce, J. Phys. A 28, 511 (1995).

[5] J. Marroquin, S. Mitter, and T. Poggio, J. Am. Stat. Assoc. 82, 76 (1987)

[6] H. Nishimori and K. Y. M. Wong, Phys. Rev. E 60, 132 (1999).

[7] K. Tanaka, Trans. Inst. Electron., Inf. Commun. Eng. A 10, 1679 (1999).
[8] D. Bolle', H. Rieger, and G. M. Shim, J. Phys. A 27, 3411 (1994).

[9] D. M. Carlucci and J. Inoue, Phys. Rev. E 60, 2547 (1999).

[10] J. Inoue and D. M. Carlucci, Phys. Rev. E 64, 036121 (2001).

[11] D. J. C. Mackay, IEEE Trans. Inf. Theory 45, 399 (1999).

[12] K. Tanaka and T. Morita, Trans. Inst. Electron., Inf. Commun. Eng. A 6, 1033 (1997).

[13] K. Tanaka, M. Ichioka, and T. Morita, Trans. Inst. Electron., Inf. Commun. Eng. A 1, 260 (1997).

[14] The standard images that are used in this paper are available at ftp://ftp.lab1.kuis.kyoto-u.ac.jp/pub/sidba/

[15] I. Pitas and A. N. Venetsanopoulos, Nonlinear Digital Filters (Kluwer Academic, Boston, 1990). 